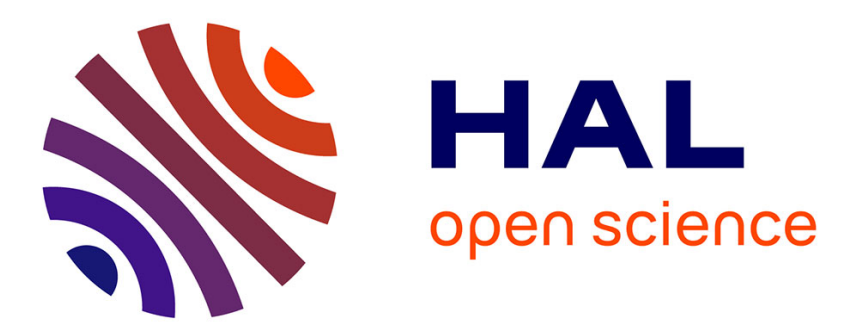

\title{
Sophorolipid production by on oils with a special fatty acid composition and their consequences on cell viability
}

\author{
I. N. A. Bogaert, S. Roelants, D. Develter, W. Soetaert
}

\section{To cite this version:}

I. N. A. Bogaert, S. Roelants, D. Develter, W. Soetaert. Sophorolipid production by on oils with a special fatty acid composition and their consequences on cell viability. Biotechnology Letters, 2010, 32 (10), pp.1509-1514. 10.1007/s10529-010-0323-8 . hal-00606294

\section{HAL Id: hal-00606294 \\ https://hal.science/hal-00606294}

Submitted on 6 Jul 2011

HAL is a multi-disciplinary open access archive for the deposit and dissemination of scientific research documents, whether they are published or not. The documents may come from teaching and research institutions in France or abroad, or from public or private research centers.
L'archive ouverte pluridisciplinaire HAL, est destinée au dépôt et à la diffusion de documents scientifiques de niveau recherche, publiés ou non, émanant des établissements d'enseignement et de recherche français ou étrangers, des laboratoires publics ou privés. 
Section: Microbial and Enzyme Technology

Sophorolipid production by Candida bombicola on oils with a special fatty acid composition and their consequences on cell viability

I . N. A. Van Bogaert ${ }^{1, *}$ S. Roelants $^{1}$, D. Develter ${ }^{2} \&$ W. Soetaert ${ }^{1}$

${ }^{1}$ Laboratory of Industrial Microbiology and Biocatalysis, Department of Biochemical and Microbial Technology, Faculty of Bioscience Engineering, Ghent University, Coupure Links 653, B-9000 Ghent, Belgium

${ }^{2}$ Ecover Belgium NV, Industrieweg 3, B-2390 Malle, Belgium

*corresponding author

Te1: +32-9-264.60.34, Fax:+32-9-264.62.31, Email: Inge.VanBogaert@UGent.be

Keywords: Candida bombicola, coconut oil, meadowfoam oil, medium-chain fatty acids, toxicity of free fatty acids, sophorolipids 


\begin{abstract}
Sophorolipids production by the yeast Candia bombicola is most favourable when glucose is used as a carbon source in combination with a hydrophobic carbon source such as a common vegetable oil. Most vegetable oils are comprised of C16-C18 fatty acids, an ideal range for sophorolipid production. The use of oils with either shorter or longer fatty acids, such has coconut oil or meadowfoam oil, respectively, was evaluated. Such oils did not contribute to enhanced sophorolipid production when compared to cultures run on glucose as the sole carbon source. Moreover, a toxic effect of medium-chain fatty acids towards stationary $C$. bombicola cells was demonstrated.
\end{abstract}

\title{
Introduction
}

Sophorolipids (Fig. 1) are biological surface active agents (biosurfactants) synthesized by a selected number of yeast species such as Candida bombicola, C. apicola, C. batistae, Wickerhamiella domericqiae and Rhodotorula bogoriensis (Spencer et al. 1970; Gorin et al. 1961; Konishi et al. 2008; Chen et al. 2006 ; Tulloch et al. 1968). The sophorolipids produced by $C$. bombicola have attracted some industrial attention due to their excellent surface lowering properties, environmental friendly profile and good fermentation yields. These molecules not only act as detergent, emulsifier or wetting agent in the various conventional surfactant sectors (cleaning, cosmetics, paints, food, etc.), but find thanks to their biological activity also potential application as antimicrobial, immunemodulating, antiviral and anticancer agent (reviewed in Van Bogaert et al. 2007).

Sophorolipids synthesized by $C$. bombicola are excreted as a mixture of slightly different molecules varying in their degree of acetylation, presence or absence of lactonization (internal esterification between the fatty acid carboxyl group and the 4' '-position of the sophorose 
molecule), position of the fatty acid hydroxyl group ( $\omega$ or $\omega-1)$, fatty acid chain length and saturation. Despite these variations, the fatty acid tail of sophorolipids is, in general, limited to 16 or 18 carbon atoms. When sophorolipids are produced on glucose as the sole carbon source (so-called de novo sophorolipid synthesis), stearic acid (C18:0) is the major component. However, higher biosurfactant yields are obtained when a second hydrophobic carbon source is added. Taking the profile of the de novo sophorolipid fatty acid tail into account, most common vegetable oils such as rapeseed, sunflower, olive, safflower, soybean and corn oil which are rich in C16-18 fatty acids - are highly suitable and are readily incorporated into the sophorolipid molecule. These vegetable oils have a low content of saturated fatty acids, and only contain stearic acid as a minor component. However, excellent results are obtained with oils rich in oleic acid such as rapeseed oil and in most cases, the fatty acid composition of the applied vegetable oil is reflected in the fatty acid pattern of the sophorolipid mixture, illustrating the direct incorporation of the substrates (Davila et al. 1994).

In the experiments described in this manuscript, we move away from the common vegetable oils and test oils with fatty acids either shorter or longer than the $\mathrm{C} 16-\mathrm{C} 18$ range. As a decrease in biomass formation was observed when coconut oil was applied, the possible cause of this event was further investigated.

[insert Figure 1]

\section{Mate rials and methods}

\section{Strains and culture conditions}

Candida bombicola ATCC 22214 was used in all experiments. The medium described by Lang et al. (2000) was applied for sophorolipid production. Shake-flask cultures $(200 \mathrm{ml}$ culture medium) were incubated at $30{ }^{\circ} \mathrm{C}$ and $200 \mathrm{rpm}$. Feeding of the hydrophobic carbon 
source was started $48 \mathrm{~h}$ after inoculation, with a total amount of $37.5 \mathrm{~g} / \mathrm{l}$ unless stated otherwise. A control without the addition of hydrophobic carbon source was run in parallel for all experiments. Coconut oil and fatty acids were obtained from Sigma. Meadowfoam oil was obtained from Natural Plant Products Inc. The fatty acid composition of meadowfoam oil was provided by the supplier. The composition of coconut oil was determined by GC-MS analysis as described below. Incubation was stopped 8 days after the first addition of hydrophobic carbon source unless stated otherwise.

\section{Biomass formation}

Biomass formation was monitored from the colony forming units (c.f.u). determined on agar plates containing $100 \mathrm{~g}$ glucose $/ 1,10 \mathrm{~g}$ yeast extract/l and $1 \mathrm{~g}$ urea/l that were incubated at 30 ${ }^{\circ} \mathrm{C}$ for three days. Samples were performed in duplicate. In case of presumed high toxicity, cell viability was also checked by light microscopy; affected cells are much smaller and thinner than normal cells and do not possess the big lipid bodies as seen for unaffected cells.

\section{Sophorolipid formation and analysis}

Analytical sophorolipid samples were prepared as follows: $440 \mu$ l ethyl acetate and $11 \mu \mathrm{l}$ acetic acid were added to $1 \mathrm{ml}$ culture broth and shaken vigorously for $5 \mathrm{~min}$. After centrifugation at $9000 \mathrm{~g}$ for $5 \mathrm{~min}$, the upper solvent layer was collected and $600 \mu \mathrm{l}$ ethanol was added to it. The sophorolipid samples were analysed by HPLC on a Varian Prostar HPLC system using a Chromolith Performance RP-18e 100-4.6 mm column from Merck KGaA at $30{ }^{\circ} \mathrm{C}$ and evaporative light scattering detection (Alltech). 


\section{Final sophorolipid extraction from culture broth and}

This protocol is based on the one described in Fleurackers et al. (2010). Three volumes of ethanol were added to the residual fermentation medium and yeast cells were removed by centrifugation. The water/ethanol mixture of the supernatants was removed by evaporation in a rotary evaporator. Two volumes of ethanol were added to dissolve the sophorolipids and the residual hydrophobic carbon source and the mixture was passed through a Whatman filter. The ethanol in the filtrate was evaporated and 1.5 volume diethyl ether was added to dissolve the residual hydrophobic carbon source and the diethyl ether mixture was transferred to a Whatman filter. The residue left in the boiling flask consists of sophorolipids. Weights of the different fractions were determined.

\section{Gas chromatography and mass spectroscopy analysis}

The fatty acid composition of coconut oil and the fatty acid part of the sophorolipids was analysed by GC and MS analysis. Prior the analysis, the fatty were split off and modified to fatty acid methyl esters (FAMEs) by acidic methanolysis.

GC-MS analysis of the FAMEs was performed with the 6890N Network GC System and the 5973 Network Mass Selective Detector from Agilent Technologies. Samples were brought on a CP-Wax $52 \mathrm{CB}$ column (Varian) by direct injection at $240{ }^{\circ} \mathrm{C}$ and a split ratio of 50:1. Helium was used as carrier gas at $1 \mathrm{ml} / \mathrm{min}$. The column oven started at $45{ }^{\circ} \mathrm{C}$ and increased to $240{ }^{\circ} \mathrm{C}$ at $10{ }^{\circ} \mathrm{C} / \mathrm{min}$. 


\section{Results and discussion}

Oils with a unusual fatty acid composition were used as hydrophobic carbon source for sophorolipid production in shake flask experiments. Coconut oil was used to test oils containing medium-chain fatty acids, whereas meadowfoam was applied to verify sophorolipid production on very-long chain fatty acids. A culture medium with high glucose content was used $(120 \mathrm{~g} / \mathrm{l})$, as it is thought that a high glucose concentration prevents fatty acids metabolization by the $\beta$-oxidation cycle (Lang et al. 2000).

\section{Meadowfoam oil}

Meadowfoam oil is pressed from the seeds of meadowfoam (Limnanthes alba) and contains over $98 \%$ fatty acids having 20 carbon atoms or more. The exact fatty acid composition is given in Table 1.

[insert Table 1]

The c.f.u. values were similar to fermentations on conventional hydrophobic carbon sources (over $9 \log$ c.f.u./ml); meadowfoam oil was not toxic to $C$. bombicola cells.

During the incubation time, the oil remained present on the culture surface and was still visible at the end of the experiment. This indicated that meadowfoam oil was not or not completely utilized as a substrate for sophorolipid production. After separation of the sophorolipids and the residual oil, it turned out that $77 \%$ of the initially added oil was still present and consequently not incorporated into sophorolipids. Furthermore, the overall sophorolipid yield was not so high (only $16.7 \mathrm{~g} / \mathrm{l}$ ) and is comparable to yields achieved for the control, i.e. a culture to which no hydrophobic carbon source was added (de novo synthesis of sophorolipids). The fatty acid composition of the meadowfoam based sophorolipids is indeed similar to the one observed for de novo sophorolipids (Table 2). 
[insert Table 2]

\section{Coconut oil}

Coconut oil is one of the rare vegetable oils rich in medium-chain fatty acids; its detailed composition is given in Table 1.

Casas and García-Orchoa (1999) are one of the few researchers who tested coconut oil as a hydrophobic carbon source. Coconut oil turned out to be inferior for sophorolipid production when compared to palmitic acid, corn oil, grapeseed oil, olive oil and sunflower oil, but no further attention was given to possible reasons of this deficiency or to characterisation of the sophorolipids. Ogawa and Ota (2000) reported similar results; sophorolipid production was worse when compared to olive, rapeseed and soybean oil, but still slightly higher compared to cultures were no hydrophobic carbon source was added, suggsting that the coconut oil contributes to sophorolipid formation. When focussing on the fatty acid composition of the biosurfactant molecules, no coconut oil derived medium-chain fatty acids were retrieved; all fatty acids were within the conventional C16-C18 range. Furthermore, biomass formation was substantially lower when compared to the other conditions (7 g/l versus $23 \mathrm{~g} / \mathrm{l}$; Ogawa and Ota 2000). We also observed this later finding when looking at c.f.u. values during our experiments when coconut oil was added to stationary cells. Yet, the effect on stationary cells was not that strong or only occurred at the end of the incubation period when all glucose was consumed. Hence, the question arose if low sophorolipid and biomass yields on coconut oil were related to toxicity of the oil to C. bombicola cells.

Toxicity of coconut oil could be explained by the fact that the yeast starts using coconut oil as carbon source and that the presence of medium-chain fatty acids - released from the coconut triacylglycerides by esterases - has a toxic effect on the yeasts cells. It is demonstrated that capric acid (10:0), lauric acid (12:0) and their 1-monoacylglycerols have not only 
microbicidal activity against enveloped viruses and various bacteria, but also against the yeast C. albicans. Even at concentrations lower than $10 \mathrm{mM}$ and short incubation times, a clear inhibitory effect was observed due to the disruption or disintegration of the plasma membrane which causes disorganisation and shrinkage of the cytoplasm (Bergsson et al. 2001). Because of the potential toxic effect of free fatty acids, we tested their effect on $C$. bombicola in the stationary phase. Fatty acids were added after $48 \mathrm{~h}$ cultivation time at $10 \mathrm{mM}$ thereby simulating the addition of the hydrophobic carbon source. The results are shown in Fig. 2. While caprylic acid (8:0) was toxic with a $100 \%$ killing of the yeast cells within $1 \mathrm{~h}$, the maximal effect of capric acid (10:0) was reached after a few hours of incubation and was not complete. For fatty acids with a chain length over 10 carbon atoms, no lethal effects were observed at $10 \mathrm{mM}$. After this first toxicity screening experiment, further experiments with different concentrations were conducted.

\section{[insert Figure 2]}

Because of the extreme effect of caprylic acid, this fatty acid was tested in lower concentrations to find the level at which no toxicity is observed. As demonstrated in Fig. 3, it turned out that $2 \mathrm{mM}$ could kill all $C$. bombicola cells after 5 h exposure, while $5 \mathrm{mM}$ had the same effect after $30 \mathrm{~min}$. Only at $1 \mathrm{mM}$, however, there was no effect after $24 \mathrm{~h}$. These results hereby confirm the severe toxicity of caprylic acid toward C. bombicola cells.

[insert Figure 3]

Also the toxicity of capric acid was investigated more in detail (Fig. 4): at $5 \mathrm{mM}$ or higher it was inhibitory. However, this effect was as drastic as that with caprylic acid. Even at $100 \mathrm{mM}$ and $24 \mathrm{~h}$ incubation there were still viable cells. When examining the killing curves, one can see that there is a resistance towards the fatty acid and that the effect of 40,70 and $100 \mathrm{mM}$ is more or less the same. Finally, the toxicity of lauric acid (14:0) was further tested (Fig. 5). 
This fatty acid has a low toxicity towards C. bombicola cells and only at 70 and $100 \mathrm{mM}$ was there a minor effect.

[insert Figure 4] [insert Figure 5]

\section{Conclusion}

The described experiments illustrate that for optimal sophorolipid production oils with a fatty acid profile similar to the one of sophorolipids are preferred (i.e. saturated and unsaturated C16-C18 fatty acids). Sophorolipid production on oils containing either shorter or longer fatty acids is suboptimal and moreover, these fatty acids are not retrieved in the sophorolipid mixture; the de novo $\mathrm{C} 16-\mathrm{C} 18$ pattern is conserved.

Furthermore, a harmful effect of coconut oil towards C. bombicola was observed and it was demonstrated that this toxic effect was caused by medium-chain fatty acids. The killing effect on the yeast cells increases when the fatty acid chain length decreases. The exact mechanism of the toxicity is not known, but it is suggested that the medium-chain fatty acids interfere with the biological membranes; on one hand they disturb the membrane potential by affecting proton and ion transport and on the other hand they physical or chemical bind to intracellular membranes in this way interfering with the function of the corresponding organelles. The effect of a specific medium-chain fatty acid cannot by superimposed from one species to antother. For instance, C. bombicola is most affected by carpylic acid while this component has no effect on $C$. albicans cells; carpic and lauric acid show the highest activities towards this later yeast. It is therefore suggested that the toxic effects are caused by the lack of specific length-dependent fatty acid transporters, resulting in the accumulation of free fatty acids in the cytoplasm. 
C. bombicola is sensitive to caprylic acid: $2 \mathrm{mM}$ can kill all cells in a fermentation medium after $5 \mathrm{~h}$. When calculating this back to coconut oil (triacylglycerols, with $7 \%$ caprylic acid), this means that a theoretical value of $4.4 \mathrm{~g}$ coconut oil/ 1 has a total lethal effect while $2.2 \mathrm{~g} / \mathrm{l}$, corresponding with $1 \mathrm{mM}$ caprylic acid, has no effect. Nevertheless, when $37.5 \mathrm{~g}$ coconut oil/1 is added to the fermentation medium, no direct and such extreme toxicitywas observed. This could be explained by the fact that caprylic acid in oil is present as part of a triacylglycerol molecule and is only released gradually as free fatty acid in the medium as a result of the activity of esterases.

\section{Acknowledgments}

The authors wish to thank Ecover Belgium NV and the Bijzonder Onderzoekfonds of Ghent University for financial support (grants A05/003 and 01D18604).

\section{References}

Bergsson G, Arnfinnsson J, Steingrimsson O, Thormar H (2001) In vitro killing of Candida albicans by fatty acids and monoglycerides. Antimicrob Agents Chemother 45:32093212

Casas JA, Garcia-Ochoa F (1999) Sophorolipid production by Candida bombicola: medium composition and culture methods. J Biosci Bioeng 88: 488-494

Chen J, Song X, Zhang H, Qu YB, Miao JY (2006) Production, structure elucidation and anticancer properties of sophorolipid from Wickerhamiella domercqiae. Enzyme Microb Technol 39:501-506 
Davila AM, Marchal R, Vandecasteele JP (1994) Sophorose lipid production from lipidic precursors - Predictive evaluation of industrial substrates. J Ind Microbiol 13:249-257

Fleuracker, SJJ, Van Bogaert INA, Develter D (2010) On the production and identification of medium-chained sophorolipids. Eur J Lipid Sci Technol doi: 10.1002/ejlt. 200900158.

Gorin PAJ, Spencer JFT, Tulloch AP (1961) Hydroxy fatty acid glycosides of sophorose from Torulopsis magnoliae. Can J Chem 39:846-855

Konishi M, Fukuoka T, Morita T, Imura T, Kitamoto D (2008) Production of new types of sophorolipids by Candida batistae. J Oleo Sci 57:359-369

Lang S, Brakemeier A, Heckmann R, Spockner S, Rau U (2000) Production of native and modified sophorose lipids. Chim Oggi-Chem Today 18:76-79

Ogawa S, Ota Y (2000) Influence of exogenous natural oils on the omega-1 and omega-2 hydroxy fatty acid moiety of sophorose lipid produced by Candida bombicola. Biosci Biotechnol Biochem 64: 2466-2468

Spencer JFT, Gorin PAJ, Tulloch AP (1970) Torulopsis bombicola sp. n. Antonie Van Leeuwenhoek 36:129-133

Tulloch AP, Spencer JFT, Deinema MH (1968). A new hydroxy fatty acid sophoroside from Candida bogoriensis. Can J Chem 46:345-348

Van Bogaert INA, Saerens K, De Muynck C, Develter D, Soetaert W, Vandamme EJ (2007) Microbial production and application of sophorolipids. Appl Microbiol Biotechnol 76: 23-34 
Table 1 Fatty acid composition of meadowfoam seed oil and coconut oil

\begin{tabular}{ccc}
\hline & \multicolumn{2}{c}{ Relative Percentage (\% w/v) } \\
\hline fatty acid & meadowfoam oil & coconut oil \\
\hline $6: 0$ & - & - \\
$8: 0$ & - & 6 \\
$10: 0$ & - & 8 \\
$12: 0$ & - & 47.5 \\
$14: 0$ & - & 17.5 \\
$16: 0$ & - & 7.5 \\
$18: 0$ & - & 2.5 \\
$18: 1$ & - & 9 \\
$18: 2$ & - & 2 \\
$18: 3$ & - & - \\
$<: 20$ & 2 & - \\
$20: 0$ & 1 & - \\
$20: 1 \Delta 5$ & 63 & - \\
$22: 1 \Delta 5$ & 4 & - \\
$22: 1 \Delta 13$ & 12 & - \\
$22: 2 \Delta 5,13$ & 17 & - \\
others & 1 & \\
\hline & & - \\
& & -
\end{tabular}


Table 2 Hydroxy fatty acid composition of de novo and meadowfoam based sophorolipids as determined by GC-MS analysis

Hydro xy acids (\%)

\begin{tabular}{lccccc} 
Hydrophobic C- source & 15-OH C16:0 & 16-OH C16:0 & 17-OH C18:0 & 17-OH C18:1 & 18-OH C18:1 \\
\hline none (glucose only) & 10 & 10.5 & 33.5 & 41.5 & 4.5 \\
meadowfoam oil & 10.5 & 11.5 & 32 & 42 & 4 \\
\hline
\end{tabular}




\section{Figure captions}

Fig.1 Examples of sophorolipids produced by $C$. bombicola; a 17-L-([2'-O- $\beta$-Dglucopyranosyl- $\beta$-D-glucopyranosyl]-oxy)-octadecenoic acid 1',4"-lactone 6',6" diacetate, diacetylated lactonic sophorolipid; b 17-L-([2'-O- $\beta$-D-glucopyranosyl- $\beta$-D-glucopyranosyl]oxy)-octadecenoic acid, non-acetylated open-chain sophorolipid

Fig. 2 Effect of $10 \mathrm{mM}$ saturated fatty acids on CFU formation after 1, 5 and 24 hours of incubation

Fig. 3 Effect of different concentrations of caprilic acid on cell viability. The results after $24 \mathrm{~h}$ were the same as those after $5 \mathrm{~h}$ and are therefore not shown in the graph.

Fig. 4 Effect of different concentrations of capric acid on cell viability

Fig. 5 Effect of different concentrations of lauric acid on cell viability. Please notice that the intercept of the $\mathrm{Y}$-axis with the $\mathrm{X}$-axis differs from the previous graphs. 
Figure 1
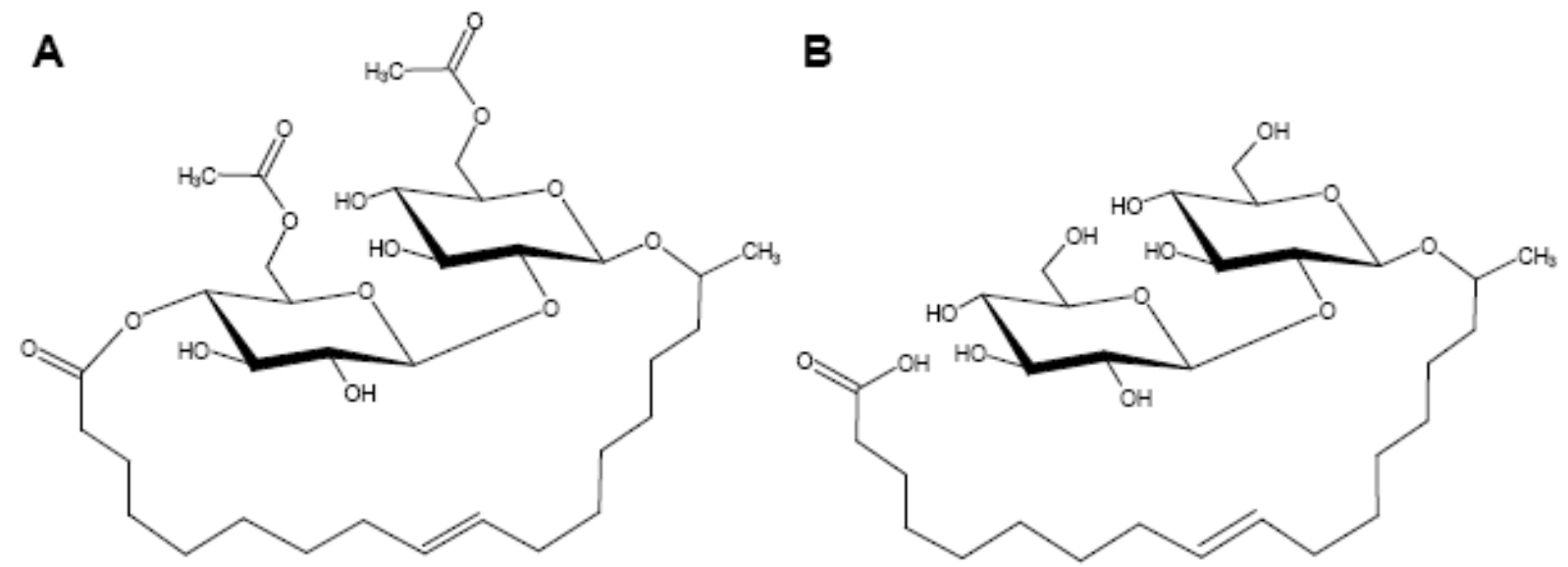
Figure 2

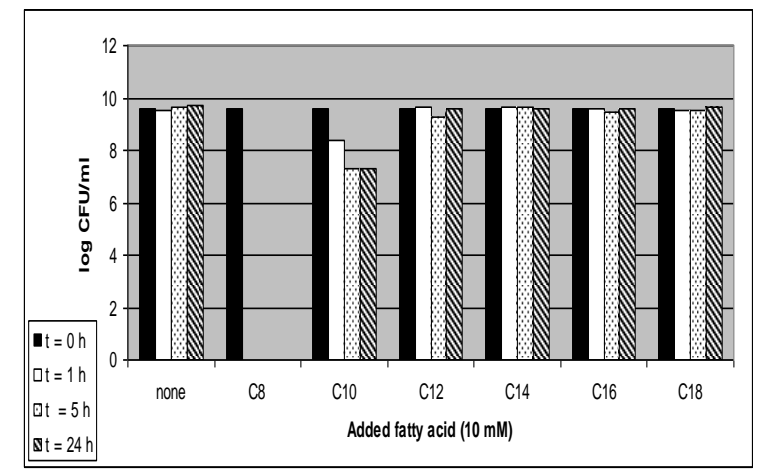


Figure 3

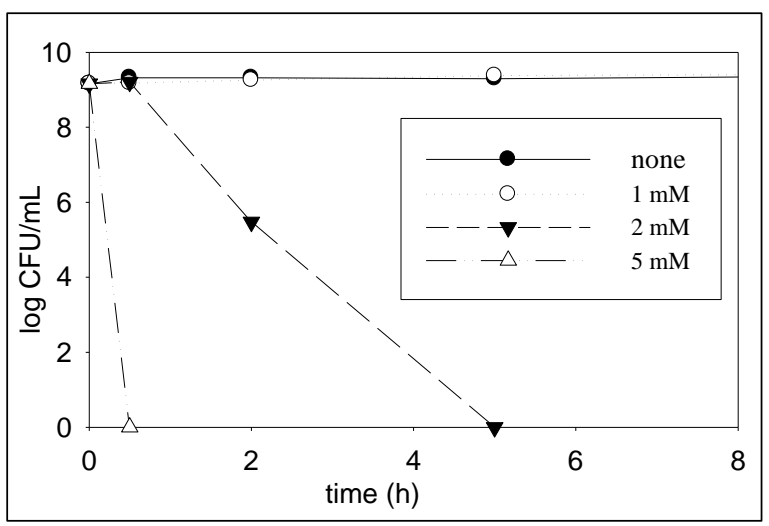


Figure 4

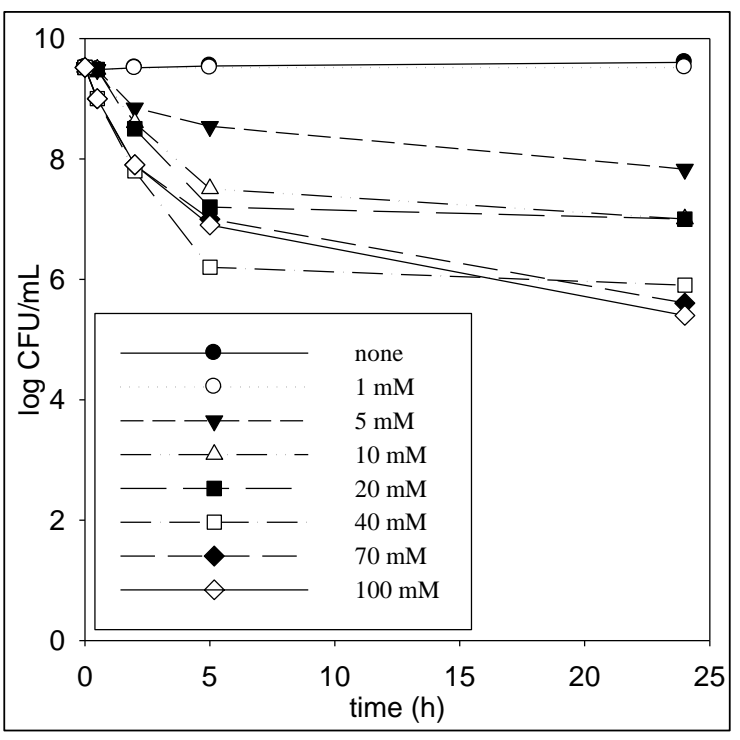


Figure 5

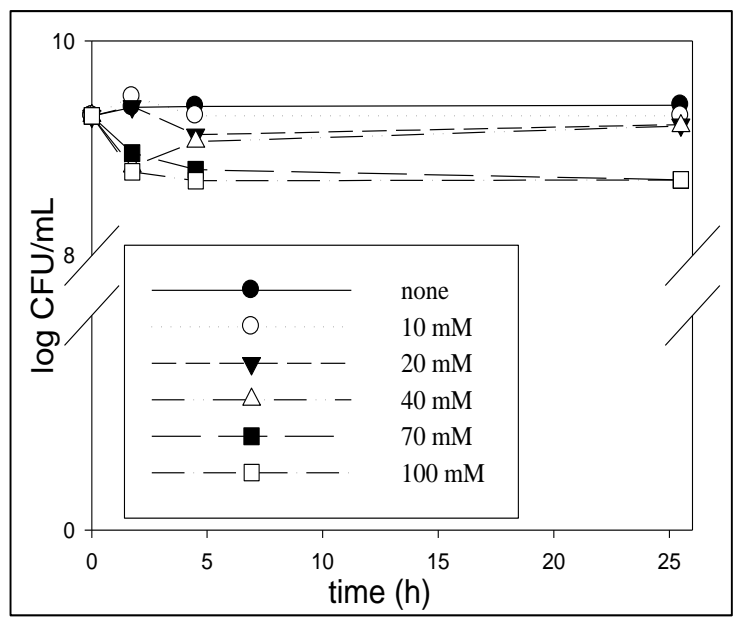

